\section{Depressive symptoms worsen outcome after MI}

Patients with acute myocardial infarction (MI) are prone to depression, and depressive symptoms have been shown to be associated with increased morbidity and mortality after MI. Hypothesizing that the time course of depression following $\mathrm{Ml}$ could affect outcome, the PREMIER Registry Investigators prospectively compared depressive symptoms at hospitalization and 1 month after discharge with 6-month outcome in 1,873 patients with MI.

Moderate to severe depressive symptoms were reported by $20.6 \%$ of patients during hospitalization, and $13.1 \% 1$ month after discharge; $7.1 \%$ of patients had depression at both time points (persistent), $13.5 \%$ had depression during hospitalization but not at 1 month (transient), 6.0\% had depression at 1 month but not during hospitalization (new), and $73.5 \%$ did not have depressive symptoms at either time point. Increasing severity of depression at baseline correlated with increased likelihood of depression at 1 month. All evaluated categories of depression increased the rate of rehospitalization and mortality combined, angina, and physical limitation, and decreased quality of life, compared with no depression. These results were found whether depression was transient, new, or persistent, although the latter two categories were associated with higher odds ratios than the first for all end points studied.

The authors conclude that depressive symptoms following $\mathrm{Ml}$ are strongly and independently associated with worse outcome, irrespective of their time course in the month after Ml. This factor had greater prognostic significance than traditional measures of disease severity and emphasizes the importance of screening for depressive symptoms at both hospitalization and 1 month after discharge.

Original article Parashar S et al. (2006) Time course of depression and outcome of myocardial infarction. Arch Intern Med 166: 2035-2043

\section{Combined antithrombotic regimens confer a higher risk of upper gastrointestinal bleeding}

With the advent of antiplatelet drugs such as clopidogrel and dipyridamole, and trial results indicating the superiority of combined drug regimens for preventing thrombosis, antithrombotic treatment regimens are becoming increasingly aggressive. To assess the risk of serious upper gastrointestinal bleeding associated with more-aggressive antiplatelet therapies, and to examine the trends in prophylactic antiplatelet treatment, Hallas et al. carried out a population-based study in Funen County, Denmark.

Among the 1,443 cases of gastrointestinal bleeding identified in Funen County during the period 2000-2004, 380 cases were exposed to at least one antithrombotic drug. Notably, use of combined antithrombotic therapy was consistently associated with a higher risk of serious upper gastrointestinal bleeding than use of a single-drug regimen (adjusted odds ratios 2.3-7.4 and 1.1-1.9, respectively). The increased risk associated with the combined regimens was synergistic - that is, more than a simple addition of the risks associated with each individual drug.

Over the period 1995-2004, the researchers documented a $425 \%$ increase in the use of combined antithrombotic regimens. Given the shift towards more-aggressive antithrombotic regimens and the higher risk of gastrointestinal bleeding that these regimens confer, the authors suggest that future trials should aim to identify subgroups of patients who are at particularly high risk of gastrointestinal bleeding. Such results would aid clinicians in the individualization of antithrombotic treatment.

Original article Hallas J et al. (2006) Use of single and combined antithrombotic therapy and risk of serious upper gastrointestinal bleeding: population based case-control study. BMJ 333: 726-731

\section{Screening program reduces sudden cardiac death in young athletes}

Young competitive athletes are at risk of sudden cardiac death (SCD) due to previously unsuspected structural heart disease. A study performed in Italy indicates that screening athletes for cardiomyopathies before sports participation, and disqualifying those with positive results, can decrease the rate of SCD substantially.

A nationwide preparticipation screening program based on 12-lead electrocardiography, patient history and examination, was 